\title{
Factores relacionados con los eventos adversos por medicamentos en niños hospitalizados en una institución de Salud en Sucre 2014-2018
}

\author{
Factors related to adverse events from medicines in children hospitalized in a \\ health institution in Sucre 2014-2018
}

\author{
Cielo Martínez Reyes ${ }^{1}$, María Horta Murcia ${ }^{2}$, Carmen Martínez Vergara ${ }^{3}$, Sandra Osorio \\ Loaiza $^{4}$, Julián Esteban Vinasco Isaza ${ }^{5}$
}

\begin{abstract}
RESUMEN
Objetivo: Determinar los factores relacionados con los eventos adversos por medicamentos en niños hospitalizados en una institución de salud de Sucre-Colombia. Material y métodos: Estudio observacional descriptivo, retrospectivo y relacional, en el que se analizaron 20 niños que tuvieron 23 Eventos Adversos por Medicamentos entre enero de 2014 y junio de 2018. Resultados. 45\% de los niños eran menores de un año de edad y $91,3 \%$ eran polimedicalizados. EI EAM más frecuente fue la flebitis química con un $34,78 \% .91,30 \%$ eran prevenibles, porque fueron consecuencia de fallas humanas como error en la prescripción (13,04\%), dispensación (13,04\%), aplicación del medicamento equivocado (30,43\%), dilución incorrecta $(13,04 \%)$, no se verificó la permeabilidad del acceso venoso $(8,7 \%)$ y $17,3 \%$ fueron EAM trazadores, es decir, se cometieron equivocaciones en varias fases del proceso. $43,47 \%$ ocurrieron en la noche. Hubo un EAM centinela y un $65,22 \%$ fueron leves. No hubo evidencia del seguimiento que hizo enfermería después del EAM en un $65,22 \%$ de los casos. Hubo relación estadística entre la prolongación de la estancia $(p=0,018)$, la prescripción $(p=0,018)$, errores de enfermería $(p=0,0023)$ y sitio anatómico afectado $(p=0,001)$ con la ocurrencia de los EAM. Conclusiones. Los EAM siempre están presentes, pues son implícitos al cuidado; su prevención depende del compromiso institucional, de los profesionales ante el correcto cumplimiento de su labor y de involucrar a los cuidadores y al mismo paciente en su seguridad.
\end{abstract}

Palabras clave: Cuidado de enfermería, pediatría, medicamentos, seguridad del paciente. Fuente (DeCS)

\begin{abstract}
Objective. To determine the factors related to adverse drug events in children hospitalized in a health institution in Sucre-Colombia. Material and methods. Observational, descriptive, retrospective and relational study, in which 20 children were analyzed who had 23 Adverse Events for Drugs between January 2014 and June 2018. Results. 45\% of the children were under one year of age and $91.3 \%$ were polyimedicalized. The most frequent EAM was the chemical phlebitis with $34.78 \% .91 .30 \%$ were preventable, because they were a consequence of human failures such as error in the prescription $(13.04 \%)$, dispensation $(13.04 \%)$, application of the wrong medication $(30.43 \%)$, incorrect dilution (13.04) \%), the permeability of the venous access was not verified (8.7\%) and $17.3 \%$ were EAM tracers, that is, mistakes were made in several phases of the process. $43.47 \%$ occurred at night.
\end{abstract}

\footnotetext{
${ }^{1}$ Corporación Universitaria Adventista, Medellín, Colombia Orcid ID: 0000-0003-1545-5094

${ }^{2}$ Corporación Universitaria Adventista, Medellín, Colombia Orcid ID: 0000-0001-8524-700X

${ }^{3}$ Corporación Universitaria Adventista, Medellín, Colombia Orcid ID: 0000-0003-4578-936X

${ }^{4}$ Corporación Universitaria Adventista, Medellín, Colombia Orcid ID: 0000-0002-4420-0536

${ }^{5}$ Corporación Universitaria Adventista, Medellín, Colombia

Orcid ID: 0000-0003-3894-2805
} 
There was a sentinel EAM and $65.22 \%$ were mild. There was no evidence of nursing follow-up after the EAM in $65.22 \%$ of the cases. There was a statistical relationship between prolongation of stay ( $p$ $=0.018)$, prescription $(p=0.018)$, nursing errors $(p=0.0023)$ and anatomical site affected $(p=0.001)$ with the occurrence of them EAM. Conclusions. EAMs are always present, because they are implicit in care; the prevention depends on the institutional commitment of the professionals before the correct fulfillment of their work and of involving the caregivers and the patient in their safety.

Keywords: Nursing care, pediatrics, medications, patient safety. Sourse (DeCS)

\section{INTRODUCCIÓN}

El cuidado, esencia de la Enfermería, es definido como "la capacidad para de aplicar tecnologías, técnicas, procedimientos, maquinarias, acciones o sencillamente hacer cosas a favor del estado de salud de nuestros pacientes ... se asocia directamente con la relación" (Riquelme, 2012) , lo cual implica el fomento de un entorno seguro (ICN, 2019). Paradójicamente, al ser una acción humana, tiene inmersa la probabilidad del error, del que derivan los eventos adversos (EA) (Martínez, 2014), más cuando se está en contacto directo con los pacientes, como ocurre con los enfermeros. Para el caso de pediatría, Achury et al (Achury, 2017)), informan que los EA más frecuentes son: úlceras por presión, flebitis, caídas, desconexión catéter central, neumonía asociada a ventilación mecánica, infección urinaria por sonda uretral y los eventos adversos por medicamentos (EAM), los cuales aportan una considerable carga (Martínez, 2019) (Gutiérrez, 2011), por lo que se deduce que cuidado directo tiene una gran cuota de responsabilidad en ellos al incumplir los estándares y no aplicar correctamente los protocolos establecidos. (Martínez, 2019) (Gutiérrez, 2011)

Hablando de los EAM, los errores más documentados que los causan son prescripción, dosis, omisión de la vía de administración y frecuencia de administración, por lo cual se puede aseverar que al menos el $50 \%$ de los EAM están relacionados con el cuidado de enfermería. (López, 2018) Esto podría ser porque "el manejo de medicamentos es un proceso complejo en el que intervienen muchas personas y diferentes profesionales, por ello hay mayor probabilidad de equivocarse" (MinSalud).

Sus consecuencias son, según Davenport et al (Davenport, 2017) la prolongación de la estancia hospitalaria, además de la afectación de la salud de los niños e incremento en el gasto de recursos institucionales, tal y como lo afirman Vergara y Fica. (Vergara, 2015) Así, la administración de medicamentos en pediatría supone un riesgo mayor, ya que la farmacocinética y farmacodinamia es diferente en el niño. Por ello, desde la Organización Mundial de la Salud (OMS), se ratifica que, "necesitamos aprender más sobre la manera en que los organismos de los niños reaccionan a los medicamentos para que podamos mejorar la salud infantil a escala mundial. Es extremadamente importante mantenerse al corriente de los posibles efectos colaterales en las poblaciones infantiles" (OMS, 2007), además de duplicar los esfuerzos para que dichos EAM disminuyan y se garantice la seguridad de los niños. (MinSalud)

En Enfermería, esto se traduce de acuerdo con las premisas de Nightingale en "estar al frente", es decir; saber cómo llevar un cargo, porque "desde la más colosal de las calamidades hasta el más pequeño de los accidentes, sus consecuencias tienen a menudo como causa la falta de quién estaba al frente, o mejor, su no saber cómo estar al frente" (Nightingale, 1990).

Por ello, cada institución de salud tiene la responsabilidad de conocer la epidemiología de sus EAM, sobre todo en pediatría y, en consecuencia, entablar planes mejoramiento efectivos. En tal sentido, la investigación tuvo como objetivo determinar los factores relacionados con los eventos adversos por medicamentos en niños hospitalizados en una institución de salud de Sucre-Colombia.

\section{METODOLOGÍA}

Se trata de un estudio observacional descriptivo, porque aborda las características del fenómeno de estudio, en este caso los EAM, sin manipular las variables, mostrando su comportamiento; es retrospectivo, porque analizaron las historias clínicas de los niños entre enero de 2014 y junio 
2018; y relacional porque se midió la probable asociación entre las variables, aportando probables indicios de causalidad. (Hernández, 2014)

\section{Muestra}

Conformada por los que tuvieron EAM entre enero de 2014 y junio de 2018.

\section{Criterios de selección}

Fueron incluidos niños entre un mes y 14 años de edad, que estuvieron hospitalizados en cualquiera de los servicios de la institución (urgencias, UCl, cirugía y hospitalización), entre 1 enero de 2014 a 30 de junio de 2018, que tuvieron EAM con información relacionada con el acontecimiento. Fueron excluidos aquellos casos cuyos expedientes estaban incompletos o se habían extraviado.

\section{Procedimiento}

Los casos analizados fueron los suministrados por la oficina de Seguridad del Paciente de la institución de salud, cuyas historias clínicas fueron leídas en su totalidad con el propósito de extraer los datos de interés, con respecto a las variables relacionadas con el paciente (intrínsecas): Edad, sexo, peso, talla, comorbilidades, historia neonatal, estancia hospitalaria, polifarmacia, motivo de consulta, diagnóstico confirmado, alergias; las relacionadas con el cuidado de enfermería y otros profesionales: errores en prescripción, dispensación, preparación y administración de los fármacos.

En los casos que luego de ser examinados hubo alguna duda con respecto a la prolongación de la estancia como consecuencia de los EAM, fueron leídas por dos expertos: un pediatra y una enfermera con experiencia y formación en farmacología.

\section{Manejo de Datos}

Inicialmente fueron organizados en una plantilla del programa Excel versión 2016, y luego exportados al programa estadístico SPSS versión 23, para aplicar medidas de estadística descriptiva. Además, se hizo un análisis bivariado, tratando de determinar relación estadísticamente significativa entre las variables independientes y la dependiente, utilizando para ello el Test de Fisher, ideal para establecer asociaciones en el caso de muestras pequeñas.

En cuanto a los aspectos éticos, se siguió la Resolución colombiana de investigación 8430 de 1993, que cataloga el estudio como sin riesgo, pues no se manipularon o intervinieron los pacientes. Además, se siguieron las directrices de la ley de protección de los datos personales (Ley 1581 de 2012), pues se mantuvo bajo reserva la identificación de los pacientes y la institución de salud que participaron en el estudio, el cual fue aprobado por el Comité de Ética de la Corporación Universitaria Adventista y de la Institución de Salud, con firma de acta de confidencialidad.

\section{RESULTADOS}

Tabla 1

Caracterización de los niños y los EAM.

\begin{tabular}{|c|c|c|c|}
\hline Variable & Categoría & Frecuencia & Porcentaje \\
\hline \multirow[t]{2}{*}{ Sexo } & Masculino & 10 & 50 \\
\hline & Femenino & 10 & 50 \\
\hline \multirow[t]{6}{*}{ Edad } & Menores de 1 año & 9 & 45 \\
\hline & De 1 a 5 años & 4 & 20 \\
\hline & De 7 a 9 años & 3 & 15 \\
\hline & De 10 a 12 años & 1 & 5 \\
\hline & Mayores de 12 años & 3 & 15 \\
\hline & 9 o menos kilos & 11 & 55 \\
\hline Peso & 10-19 kilos & 2 & 10 \\
\hline
\end{tabular}




\begin{tabular}{|c|c|c|c|}
\hline & $20-29$ kilos & 1 & 5 \\
\hline & $30-39$ kilos & 4 & 20 \\
\hline & $40-50$ kilos & 2 & 10 \\
\hline & Un Evento Adverso & 12 & 60 \\
\hline \multirow[t]{2}{*}{ Número de EA por niño } & Dos Eventos Adversos & 7 & 35 \\
\hline & Cinco Eventos Adversos & 1 & 5 \\
\hline \multirow[t]{11}{*}{ EAM } & Administración de medicamento incorrecto & 5 & 21,74 \\
\hline & Administración de dosis incorrecta & 1 & 4,35 \\
\hline & Dispensación tardía/incorrecta/medicamento no disponible & 1 & 4,35 \\
\hline & Dilución incorrecta & 1 & 4,35 \\
\hline & Quemadura de III grado en la mano & 1 & 4,35 \\
\hline & Flebitis química & 8 & 34,78 \\
\hline & $\begin{array}{l}\text { RAM por velocidad de infusión/Administración de medica- } \\
\text { mento del cual se sabe que el niño es alérgico }\end{array}$ & 1 & 4,35 \\
\hline & Omisión de medicamento & 2 & 8,70 \\
\hline & Prescripción incorrecta & 2 & 8,70 \\
\hline & Aplicación por vía y presentación incorrectas & 1 & 4,35 \\
\hline & Quemadura de III grado en la mano & 1 & 4,35 \\
\hline \multirow[t]{2}{*}{ EAM Trazador } & No & 19 & 82,7 \\
\hline & $\mathrm{Si}$ & 4 & 17,3 \\
\hline \multirow[t]{3}{*}{ Error de Clasificación } & No & 14 & 60,87 \\
\hline & $\mathrm{Si}$ & 5 & 21,74 \\
\hline & No Clasificado & 4 & 17,39 \\
\hline \multirow[t]{3}{*}{ Jornada } & Mañana & 6 & 26,08 \\
\hline & Tarde & 7 & 30,43 \\
\hline & Noche & 10 & 43,47 \\
\hline \multirow[t]{3}{*}{ Severidad } & Leve & 15 & 65,22 \\
\hline & Moderada & 6 & 26,09 \\
\hline & Grave & 2 & 8,70 \\
\hline \multirow[t]{2}{*}{ EAM Centinela } & No & 22 & 91,3 \\
\hline & $\mathrm{Si}$ & 1 & 4,30 \\
\hline \multirow[t]{2}{*}{ Prevenible } & No & 2 & 8,70 \\
\hline & $\mathrm{Si}$ & 21 & 91,30 \\
\hline \multirow[t]{4}{*}{ Protocolo de canalización } & No & & -- \\
\hline & $\mathrm{Si}$ & & -- \\
\hline & No Registra & 10 & 43,48 \\
\hline & No aplica & 13 & 56,52 \\
\hline \multirow[t]{4}{*}{ Cuidados de enfermería catéter } & Ninguno & 12 & 52,17 \\
\hline & Salinización/Permeabilización & -- & -- \\
\hline & 11 Correctos & -- & -- \\
\hline & Valoración & -- & -- \\
\hline \multirow[t]{6}{*}{ Sitio anatómicamente afectado } & No registra & -- & -- \\
\hline & Extremidades superiores & 7 & 30,43 \\
\hline & Extremidades inferiores & 3 & 13,04 \\
\hline & Piel (habones/prurito/rash) & 3 & 13,04 \\
\hline & Sistema nervioso & 3 & 13,04 \\
\hline & Sistema circulatorio & 1 & 4,35 \\
\hline $\begin{array}{l}\text { Servicio en el que ocurre el } \\
\text { evento }\end{array}$ & Desequilibrio electrolítico & 1 & 4,35 \\
\hline
\end{tabular}




\begin{tabular}{|c|c|c|c|}
\hline & No aplica & 5 & 21,73 \\
\hline & Cirugía & 2 & 8,70 \\
\hline & Internación & 7 & 30,43 \\
\hline \multirow[t]{3}{*}{ Afiliación al sistema de salud } & $\mathrm{UCl}$ & 12 & 52,17 \\
\hline & Urgencias & 2 & 8,70 \\
\hline & Vinculado & -- & -- \\
\hline \multirow[t]{5}{*}{ Motivo de consulta } & Subsidiado & 19 & 82,61 \\
\hline & Contributivo & 4 & 17,39 \\
\hline & Afecciones de sistema respiratorio & 10 & 43,48 \\
\hline & Afecciones del sistema nervioso & 4 & 17,39 \\
\hline & Afecciones del sistema osteomuscular & 1 & 4,35 \\
\hline \multirow[t]{3}{*}{ Diagnóstico confirmado } & Afecciones del sistema genitourinario & 2 & 8,70 \\
\hline & Compromiso de varios sistemas & 6 & 26,09 \\
\hline & Sistema respiratorio & 7 & 30,43 \\
\hline \multirow[t]{2}{*}{ Comorbilidad } & Sistema nervioso & 2 & 8,70 \\
\hline & Compromiso de varios sistemas, incluida la desnutrición & 14 & 60,87 \\
\hline \multirow{2}{*}{$\begin{array}{l}\text { Prolongación de la estancia } \\
\text { por el EAM }\end{array}$} & No & 11 & 52,17 \\
\hline & Sí & 12 & 47,83 \\
\hline \multirow{2}{*}{$\begin{array}{l}\text { Polifarmacia (más de } 4 \text { medi- } \\
\text { camentos) }\end{array}$} & No & 20 & 86,95 \\
\hline & Si & 3 & 13,04 \\
\hline \multirow{4}{*}{$\begin{array}{l}\text { Antibióticos formulados y apli- } \\
\text { cados }\end{array}$} & No & 2 & 8,7 \\
\hline & Si & 21 & 91,3 \\
\hline & No & 1 & 4,35 \\
\hline & Betalactámicos & 5 & 21,74 \\
\hline \multirow[t]{2}{*}{ Antiviral } & Síntesis Folatos & 1 & 4,35 \\
\hline & Lincosamidas & 2 & 8,70 \\
\hline \multirow[t]{2}{*}{ Antiemético } & No & 21 & 91,30 \\
\hline & Sí & 2 & 8,70 \\
\hline \multirow[t]{4}{*}{ AINES } & No & 18 & 78,26 \\
\hline & Sí & 5 & 21,74 \\
\hline & No & 3 & 13,04 \\
\hline & Acetaminofén & 6 & 26,09 \\
\hline \multirow[t]{2}{*}{ Antihistamínicos } & Dipirona & 6 & 26,09 \\
\hline & Más de un AINES & 8 & 34,78 \\
\hline \multirow[t]{4}{*}{ Líquidos endovenosos } & No & 21 & 91,30 \\
\hline & Uno & 2 & 8,70 \\
\hline & No & 5 & 21,74 \\
\hline & Líquidos sin electrolitos & 9 & 39,13 \\
\hline \multirow[t]{4}{*}{ Corticoides } & Líquidos con electrolitos & 6 & 26,09 \\
\hline & Líquidos con y sin electrolitos & 3 & 13,04 \\
\hline & No & 10 & 43,48 \\
\hline & Uno & 10 & 43,48 \\
\hline \multirow[t]{3}{*}{ Medicamentos implicados } & Más de uno & 3 & 13,04 \\
\hline & Betaláctamicos & 3 & 13,04 \\
\hline & Glucopeptídicos & 2 & 8,70 \\
\hline
\end{tabular}




\begin{tabular}{|c|c|c|c|}
\hline & AINES & 1 & 4,35 \\
\hline & LEV & 2 & 8,70 \\
\hline & Corticoides IV & 4 & 17,39 \\
\hline & Medicamentos inhalados & 4 & 17,39 \\
\hline & Líquidos más medicam IV & 2 & 8,70 \\
\hline & Más de un medica IV & 1 & 4,35 \\
\hline \multirow[t]{3}{*}{ Alergia } & Otros & 4 & 17,39 \\
\hline & No & 18 & 78,26 \\
\hline & $\mathrm{Si}$ & 5 & 21,74 \\
\hline \multirow[t]{2}{*}{ Error en la prescripción } & No Registra & -- & -- \\
\hline & No & 20 & 86,96 \\
\hline \multirow[t]{2}{*}{ Error en la dispensación } & $\mathrm{Si}$ & 3 & 13,04 \\
\hline & No & 20 & 86,96 \\
\hline \multirow[t]{2}{*}{ Medicamento incorrecto } & $\mathrm{Si}$ & 3 & 13,04 \\
\hline & No & 16 & 69,57 \\
\hline \multirow[t]{2}{*}{ Vía Incorrecta } & $\mathrm{Si}$ & 7 & 30,43 \\
\hline & No & 22 & 95,65 \\
\hline \multirow[t]{4}{*}{ Verificación de la permeabilidad } & $\mathrm{Si}$ & 1 & 4,35 \\
\hline & No & 2 & 8,70 \\
\hline & $\mathrm{Si}$ & 20 & 86,96 \\
\hline & N/A & 1 & 4,35 \\
\hline \multicolumn{4}{|l|}{ Error en la dilución } \\
\hline & No & 7 & 30,43 \\
\hline & $\mathrm{Si}$ & 3 & 13,04 \\
\hline & No Registra & 12 & 52,17 \\
\hline \multirow{3}{*}{$\begin{array}{l}\text { Seguimiento del niño después } \\
\text { del EAM por parte de enfermería }\end{array}$} & $\mathrm{N} / \mathrm{A}$ & 1 & 4,35 \\
\hline & No & 15 & 65,22 \\
\hline & $\mathrm{Si}$ & 8 & 34,78 \\
\hline
\end{tabular}

Tabla 2

Asociaciones estadísticas entre variables con el Test de Fisher

$\begin{array}{llll}\text { Variables independientes } & \text { Característica } & \text { EAM } & \text { F } \\ \text { Sitio de Afectación } & \text { Extremidades superiores } & 7 & 0,001 \\ & \text { Extremidades inferiores } & 3 & \\ & \text { No Aplica } & 13 & 0,018 \\ \text { Prolongación de la estancia por el EAM } & \text { No } & 20 & \\ & \text { Sí } & 3 & \\ \text { Líquidos más medicam IV } & 2 & 0,018 \\ \text { Alergia } & \text { Más de un medica IV } & 1 & \\ \text { Error de prescripción } & \text { Otros } & 4 & 0,0023 \\ \text { Error de enfermería en cuanto al me- } & \text { No } & 20 & 3 \\ \text { dicamento } & \text { Sí } & 16 & \\ & \text { Sí } & 7 & \end{array}$




\section{DISCUSIÓN}

Se hallaron 20 niños que habían tenido 23 EAM, lo que significa que hay algunos que tuvieron más de un EAM. La mitad de los niños, eran menores de un año de edad, situación que difiere con el estudio de Rodríguez et al (Rodríguez, 2017) y de Teles et al (Teles, 2019) en los que los afectados tienen edades cercanas o menores a cinco años. En lo que se refiere al sexo, los estudios. Teles et al (Teles, 2019), encontraron que los varones estuvieron presentes en un $60 \%$, de los casos, mientras que Fajreldines et al (Fajreldines, 2019), verificó que correspondía a un $48,43 \%$ y en Sucre, la mitad eran de sexo masculino, por tanto, como afirma Rodríguez (Rodríguez, 2017) no hubo predominio importante entre géneros. Haciendo un abordaje de las características de los EAM, Eshetie et al (Eshetie, 2015) informan que el que más se presentó fue la flebitis, con un $22.4 \%$ de los casos, superado por el hallado en la clínica colombiana, donde fue del $34,78 \%$, porque los fármacos que más recibieron los pacientes fueron antibióticos intravenosos, los cuales tienen una osmolaridad que puede lesionar los vasos si no se realizan las diluciones adecuadas y no se administran en el tiempo necesario. También se identificaron fallas en la aplicación de medicamento, dosis, al igual que en la investigación de Woo (Woo, 2015), donde el error de medicación ocurrió en un $41 \%$, mientras que el medicamento incorrecto en un $21,74 \%$.

Lo anterior se traduce en la necesidad de implementar estrategias que incentiven la adherencia a la aplicación de los correctos y rondas de seguridad por parte de quienes manipulan los fármacos, comunicar los errores que se cometen y fortalecer las auditorías directas a cargo de los profesionales de enfermería, más cuando los resultados revelan que el $40 \%$ de los niños sufrieron dos y hasta cinco eventos adversos además de los EAM. No obstante, este porcentaje es más alentador que el observado por Feleke et al (Feleke, 2015), quienes determinaron que, de 263 pacientes, el $56,7 \%$ sufrió más de tres tipos de errores en la administración de medicamentos. Los eventos trazadores, ocurrieron en un $17,3 \%$, pues como recalca Piñero, "es extraño que el error sea consecuencia del descuido o imprudencia de una sola persona" (Piñero, 2014). Por ello, resulta necesario reforzar que enfermería, al ser el último eslabón en la cadena de manipulación de los fármacos, se constituye en una barrera trascendental para evitar los EAM, por tanto, su gestión, supervisión y evaluación continuos durante la transcripción, preparación y aplicación de los medicamentos puede constituirse en la diferencia entre la vida y muerte del paciente. Saber cómo estar al frente, premisa promulgada por Nightingale (Nightingale, 1990), es vital para la seguridad de los niños que se cuidan.

En cuanto a su severidad, el $35 \%$ de los EAM, fueron graves o moderados, lo que representa afectación en la salud del infante y pérdidas monetarias para la institución. Los leves fueron el $65.21 \%$ de los casos, porcentaje superado por los hallazgos de Stacey et al (Stacey, 2014), donde el $80 \%$ se consideraron menores. Esta situación no debe crear una falsa seguridad y tranquilidad en los sistemas de salud, pues como lo asevera Piñero, el hecho de que un error no haya tenido consecuencias no quiere decir que el siguiente no las pueda tener, (Piñero, 2014) como en el caso de los EA centinela. A propósito de ellos, Martínez-Ozuna et al (Martínez, 2018) identificaron que $4.34 \%$ de los EAM lo eran. Entre tanto, Valdés y Duarte (Valdés, 2017) reportaron en su estudio que en Colombia el $5 \%$ de los casos de EAM terminan en consecuencias fatales como la muerte, informando que Sucre es el cuarto departamento que más los reporta. No obstante, los EAM pueden prevenirse. De hecho en este estudio el $91,3 \%$ pudieron evitarse, porcentaje similar al referido por Achury $(94,7 \%)$ (Achury, 2017), porque fueron ocasionados por "una alteración en el cumplimiento de los estándares del cuidado".(Achury, 2017)(Rendón, 2019) Es por eso que Suntasig (Suntasig, 2015) recomienda que se desarrollen programas multidisciplinarios que permita la participación del equipo de salud, donde se involucren todos para detectar, reportar y disminuir los errores, a través de la regulación de políticas respecto a la evaluación, selección y uso de fármacos, capacitación del personal al respecto, comunicación efectiva y protocolos claros donde se evidencien las concentraciones de fármacos estándar y tablas de dosificación, (MinSalud) y la notificación oportuna (Campos, 2012), que para el caso de la presente investigación se hizo en el $61 \%$ de los casos, lo cual indica que hace falta un camino considerable para lograr la cultura de la seguridad.

El servicio en el que más se registró EAM fue la Unidad de Cuidados Intensivos pediátrica, porque es un área de alta complejidad, donde hay niños graves, pluripatológicos 
y polimedicalizados, sometidos a múltiples tratamientos y procedimientos invasivos, que los tornan aún más vulnerable a los EAM. (Sakuma, 2014) A propósito del tiempo de hospitalización, los EAM ocurrieron en niños con menos de siete días de internación, lo cual concuerda con otros estudios, donde la media de hospitalización osciló entre cinco y siete días (Ji, 2018) (Riquelme, 2013). Sin embargo, discrepa de lo afirmado por autores de Brasil y Etiopía, quienes calcularon que estuvo ocho y nueve días (Teles, 2010 (Eshtie, 2019), y con Rodríguez et al (Rodríguez, 2017), quienes determinaron que la media de días de estancia intrahospitalaria en la que se registraron EAM fue de 11,4 \pm 8 días. Ante esta realidad es importante acotar que, un día más en el hospital, representa 24 horas más de exposición su ambiente, intervenciones y con frecuencia más medicamentos, por tanto, más riesgo a un sin número de factores que pueden desencadenar un EAM o cualquier otro EA. Con respecto a este análisis, Riquelme y Ourcilleón argumentan que "por cada actividad o proceso que se incorpora a la atención de un paciente, la posibilidad de que ocurra un EA se incrementa en un 1\%" (Riquelme, 2013). En lo que concierne a los medicamentos implicados, en el presente estudio fueron los antibióticos $(21,74 \%)$, esto mismo lo afirman Monroy y Nagles (Monroy, 2014) con un 40,76\% de los EAM; asimismo en la revisión hecha por Martínez et al. (Martínez, 2018). Porcentajes aún más elevados, es decir, $72 \%$, devela Eshetie (Eshetie, 2019).

En cuanto a las consecuencias producidas por los EAM, la prolongación de la estancia no solo ocurrió en un $15 \%$ de los casos, sino también tuvo una asociación estadística significativa $(p=$ 0.0018 ), la cual también fue encontrada por Fajreldines (Fajreldines, 2019), quien además pudo determinar que en promedio pueden ser 3,14 días más de internación, a consecuencia de los EAM, lo que se traduce en mayor costo económico. Tal situación es consecuencia de lesiones físicas al paciente, como es el caso de la flebitis química, tal y como se demostró en la clínica estudiada y por Milutinović et al (Milutinović, 2015) Otros aspectos analizados fueron los factores intrínsecos del niño que pudieron haber propiciado los EAM. Desde el punto de vista estadístico no se hallaron asociaciones significativas, pero es imperativo recalcar que, Eshetie et al (Eshetie, 2019) y Martínez et al (Martínez, 2014), evidenciaron que los EAM son más comunes en los primeros años de la vida. De hecho, es sabido que el paciente menor de un año de edad, tiene una actividad metabólica reducida, por lo que acumulará más medicamento y probablemente ese pequeño paciente se intoxique (Gonzáles, 2016). En los que el peso no supera los 10 kilos, como en el caso de lo hallado en la clínica colombiana, es decir $55 \%$ y en el estudio de Suntasig (Suntasig, 2015), donde el $81.4 \%$ de los niños estaban en esta condición, hay mayor cantidad de agua corporal, y, por tanto, mayor volumen aparente de distribución del fármaco, cuyo comportamiento se afecta cuando son solubles en agua. Lo mismo ocurre con la excreción de la mayoría de los fármacos, porque los procesos de secreción tubular también podrían estar inmaduros y normalizarse al año de vida. (Gonzáles, 2016) Otro hecho que puede constituirse en un riesgo para los niños es la polifarmacia, que estuvo presente en el $91,30 \%$ de los casos, principalmente con antibióticos y analgésicos. En este sentido, Woo et al (Woo, 2015) en Corea encontraron también que esta condición era muy común en los pacientes que presentaban EAM, con una mediana de 21,0 medicamentos durante su tratamiento. Ante este panorama, el conocer y capacitarse sobre la acción farmacológica, dosis, interacciones y reacciones adversas de los medicamentos de uso más frecuente en pediatría, se constituye en una obligación del personal de enfermería que habitualmente labora en estos servicios y el profesional debe velar porque esto se cumpla. Entre tanto, con respecto a los factores extrínsecos, la jornada laboral podría ser uno de ellos, porque se pudo evidenciar que la mayoría de los EAM (44\%) ocurrieron en la noche y $26 \%$ en la mañana, lo que concuerda con lo observado por Martínez-Ozuna et al (Martínez, 2018), pues en su estudio, un $36.9 \%$ de EA se dieron en la noche y un $34.8 \%$, en la mañana. De manera similar, Achury et al (Achury, 2017) hallaron que el 39,6 \% de estos EA se presentó en horario nocturno. Esto se puede relacionar con los resultados de carga laboral en la investigación realizada por Cuadros et al (Cuadros, 2017), en donde el autor analiza que el trabajo aumenta en las horas de la noche; de hecho, en el servicio de pediatría se evidenció en la asignación de 4,8 a 5,0 pacientes por enfermera en el turno de día, mientras que en el turno de noche aumentó de 6,4 a 7,0 .

En cuanto a las intervenciones de enfermería, los EAM pueden deberse a que como se corroboró en la investigación a través de análisis de las historias clínicas, no se siguen 
las pautas establecidas en protocolos como la tabla de estabilidad del servicio farmacéutico para la preparación y aplicación de los fármacos intravenosos, así como lo explican López et al en su revisión de literatura, en la que detectaron que el incumplimiento de políticas y procedimientos, en la preparación de medicamentos y en la velocidad de administración, fueron causa de error. (Soto, 2016) En este sentido se debe recalcar que, los antibióticos y otros medicamentos de uso intravenoso, requieren de una preparación que implica varios pasos: determinación de la dosis a extraer de la presentación utilizando operaciones matemáticas básicas, reconstitución y/o dilución, calcular la velocidad de infusión, cuando no hay disponibilidad de bombas de infusión, seleccionar el reconstituyente y diluyente indicados, aspectos que muchas veces son desconocidos $\mathrm{u}$ omitidos por quienes se encargan de hacerlo. Rodríguez et al (Rodríguez, 2017), identificaron que la gran mayoría de los EAM fueron consecuencia de fallas en la administración de los fármacos, y concluyen que éstos pueden ser corregidos, puesto que están estrechamente ligados con el cuidado de enfermería, lo cual es corroborado por los hallazgos del presente estudio, en el que las equivocaciones estuvieron asociadas principalmente a la administración del medicamento, dilución y vía incorrecta incorrectas, similar a establecido por Soto et al (Soto, 2016), quien afirma que los principales errores que se comenten son: medicamento, dosis, vía, dilución y tiempo de infusión incorrectos; resaltó que el $4,6 \%$ de los casos hubo errores de cálculo en la dilución y en un $37,93 \%$ falta de precisión; y por Rodríguez et al (Rodríguez, 2017) quienes hallaron que la dosis equivocada se presentaba en un $37,8 \%$ de los errores, la vía incorrecta en un $5.4 \%$, paciente incorrecto $5.4 \%$ y medicamento incorrecto $8.1 \%$.

Otro aspecto relevante es la permanencia de los catéteres venosos periférico, pues según lo establecido en este estudio, a mayor número de días con ellos, mayor probabilidad de desarrollar flebitis $(p=0.028)$, premisa que reafirman Milutinović et al (Milutinović, 2015), pues, las concentraciones muy altas de medicamentos hiperosmolares o acídicos, como lo son la gran mayoría de antibióticos (medicamentos con alto índice de formulación en el estudio), y la velocidad y volúmenes de infusión inapropiados (Milutinović, 2015), además de, el no verificar la permeabilidad del acceso venoso, como se halló en la investigación, produce una reacción irritante de la íntima del vaso sanguíneo. (Valdés, 2017) En consecuencia, es imperativo estandarizar esta parte del proceso, a través del uso de la tabla de estabilidad de los medicamentos, cuya aplicación no se evidencia en las historias clínicas revisadas de la institución estudiada, sólo en un $10 \%$ de los casos. No solo enfermería está involucrada en la cadena del error, también, aunque en menor proporción, lo están otros miembros del equipo de salud. Rodríguez et al (Rodríguez, 2017) revelaron en su investigación que el $8.1 \%$ fueron a causa de la prescripción médica, dato que coincide con la asociación demostrada en la clínica sucreña $(p=0.0018)$.

Ante los anteriores hallazgos, se puede concluir que fueron pocos los EAM reportados en los años analizados en estudio, lo que no significa que no hayan ocurrido más, sino que se reportaron menos, tal vez no de manera intensional, sino como consecuencia del desconocimiento que aún existe sobre qué se debe informar. La mayoría los EAM ocurridos son prevenibles, porque son consecuencias de fallas en el proceso de manipulación de los fármacos, como la prescripción y aplicación del medicamento, que demostraron asociación estadísticamente significativa con los EAM, los cuales, tienen consecuencias directas sobre la salud de los niños, afectando órganos como la piel y prolongando su estancia. Los menores de un año y la polifarmacia, estuvieron presentes en un porcentaje considerable, por tanto, si estas dos variables convergen, se incrementa el riesgo de EAM. Los EAM siempre están presentes, pues son implícitos al cuidado, por tanto, su prevención depende del compromiso institucional, de los profesionales ante el correcto cumplimiento de su labor y de involucrar a los cuidadores y al mismo paciente en su seguridad.

Declaración de financiamiento y de conflictos de interés:

El estudio fue financiado por los autores, quienes declaran no tener conflictos de interés

\section{Correspondencia}

Cielo Martínez Reyes

Correo electrónico: rebeca@unac.edu.co

María Horta Murcia

Correo electrónico: mfhorta@unac.edu.co 
Carmen Martínez Vergara

Correo electrónico: camartinez@unac.edu.co

Sandra Osorio Loaiza

Correo electrónico: slosorio@unac.edu.co

Julián Esteban Vinasco Isaza

Correo electrónico: jevinasco@unac.edu.co

\section{REFERENCIAS BIBLIOGRÁFICAS}

Alva, L. (2018). Espiritualidad y estilo de vida en estudiantes de Medicina Humana de la Universidad Peruana Unión, Lima, 2017. ( Tesis de maestria, Universidad Peruana Union). Recuperado de: http://repositorio.upeu.edu.pe/bitstream/ handle/UPEU/983/Lea_Tesis_Maestría_2017. pdf? sequence $=1 \&$ isAllowed $=y$

Barbosa, S., \& Urrea, Á. (2018). Influencia del deporte y la actividad física en el estado de salud físico y mental: una revisión bibliográfica. Revista Katharsis, 1(25), 1-6. Recuperado de: https://doi. org/10.25057/25005731.1023

Benites, N., Cabrera, R., \& Quezada, R. (2017). Condiciones de trabajo y salud de los conductores de mototaxis en el distrito de San Martin de Porres.(Tesis de licenciatura, Universidad Cayetano Heredia). Recuperado de: http:// repositorio.upch.edu.pe/bitstream/handle/ upch/962/Condiciones_BenitesCueva_Noemi. pdf?sequence $=1$ \&isAllowed $=y$

Bringas, C. (2004). Memoria del primer encuentro nacional de Promoción de la Salud: Jesús María, municipalidad distrital que fomenta estilos de vida saludables. Lima.Ministerio de Salud Recuperado de: http://bvs.minsa.gob.pe/local/ PROMOCION/155_ennac.pdf

Cacua, L., Carvajal, H., \& Hernández, N. (2017). Condiciones de trabajo y su repercusión e Achury,

Rodríguez, S. Díaz, J. Gómez, J. Gómez, A. Díaz, E (2017) "Caracterización de los eventos adversos reportados por enfermería en unidades de cuidado intensivo en Bogotá (Colombia)". Revista cientiofica salud uninorte., 33(2): 105-117. Recuperado de: http://rcientificas.uninorte.edu.co/ index.php/salud/article/viewArticle/8927/11192

Campos, S. Sasbón, J. Dessauer. Von, B (2012) "Los cuidados intensivos pediátricos en Latinoamérica". Medicina Intensiva. Sociedad Española de Medicina Intensiva, 36(1): 3-10.Recuperado de: http://scielo.isciii.es/scielo.php?script=sci_arttext\& pid=S0210-56912012000100002

Consejo Nacional de Enfermeras [internet] Geneve: Antistatique. ICN - International Council of Nurses. [citado 27 de febrero de 2019]. Disponible
.Recuperado de: https://www.icn.ch/es/politica-deenfermeria/definiciones

Cuadros, C. Grillo, K. Toffoletto, M. Roldán, C. Canales, M (2017) "Ocurrencia de Incidentes de Seguridad del Paciente y Carga de Trabajo de Enfermería". Revista LatinoAmericana. Enfermagem. Universidad de Sao Paulo, 25:e2841 :2-8 Recuperado de: http://www.scielo.br/scielo.php?pid=S010411692017000100319\&script=sci_arttext\&tIng=es

Davenport, C. Domínguez, P. Ferreira, J. Kannemann, A. Paganini, A. Torres F (2017) "Detección de eventos adversos en pacientes pediátricos hospitalizados mediante la herramienta de disparadores globales Global Trigger Tool". Archivos argentinos de pediatría. Sociedad Argentina de Pediatría,15( 4 ): 357-363. Recuperado de: https://www.researchgate.net/ publication/318318177_Measuring_adverse_ events_in_pediatric_inpatients_with_the_Global_ Trigger_Tool

Eshetie, T. Hailemeskel, B. Mekonnen, N. Paulos, G. Mekonnen, A. Girma, T (2015) "Adverse drug events in hospitalized children at Ethiopian University Hospital: a prospective observational study". Biomed Central Pediatrics. Springer Nature, 15(83): 2-8. Recuperado de:https://www. ncbi.nlm.nih.gov/pubmed/26173560

Evaluación de la frecuencia de eventos adversos y monitoreo de aspectos claves relacionados con la seguridad del paciente. [Internet] Colombia: Minsalud. [Consultado 12 junio 2019] Recuperado de: : https://www.minsalud.gov.co/sites/rid/Lists/ BibliotecaDigital/RIDE/DE/CA/Evaluar-frecuenciaeventos-adversos.pdf

Fajreldines, A. Schnitzler, E. Torres, S. Panattieri, N (2019) "Medición de la incidencia de eventos adversos atribuidos a la atención sanitaria en un Departamento de Pediatría de un hospital universitario". Archivos Argentinos de Pediatría. Sociedad Argentina de Pediatría, 2:e106-109. Recuperado de: https://www.sap.org.ar/docs/ publicaciones/archivosarg/2019/v117n2a11.pdf

Feleke, S. Abebe, M. Seyoum, Y (2015) "Medication administration error: magnitude and associated factors among nurses in Ethiopia". Biomedical Central Nursing. Springer Nature, 14(1): 2-8. Recuperado de: https://bmcnurs.biomedcentral. com/articles/10.1186/s12912-015-0099-1

González, C. (2016) "Farmacología del paciente pediátrico". Revista Médica Clínica Los Condes. Clínica los Condes, 27(5), 652-659. Recuperado de: https://www.elsevier.es/es-revista-revista-medicaclinica-las-condes-202-articulo-farmacologia-delpaciente-pediatrico-S0716864016300918 
Gutiérrez S, MogniA, BerónA, Iramain R (2011) “Errores de medicación en niños hospitalizados". Archivos pediátricos uruguayos. Sociedad Uruguaya de Pediatría, 82(3): 133- 140.Recuperado de: http://www.scielo.edu.uy/scielo.php?pid=S1688$12492011000300002 \&$ script=sci_arttext

Hernandéz, R. Fernandéz, C. Baptista, P. (2014). Metodología de la investigación. México: McGraw-Hill education. Recuperado de: https://www.esup.edu.pe/descargas/ dep_investigacion/Metodologia $\% 20$ de $\% 20$ la $\% 20$ investigaci $\%$ C 3 \% B 3 n 205 ta $\% 20$ Edici\%C3\%B3n.pdf

Ji, H. Song, L. Xiao, J. Guo, Y. Wei, P. Tang, T (2018) "Adverse drug events in Chinese pediatric inpatients and associated risk factors: a retrospective review using the Global Trigger Tool. Scientific Reports. Nature, 8(2573): 1-7. Recuperado de: https://www. nature.com/articles/s41598-018-20868-2

La salud es de todos. [internet] Bogotá: minsalud. [consultado 24 feb 2019] Recuperado de: https:// www.minsalud.gov.co/salud/publica/poblacionesvulnerables/paginas/poblaciones-vulnerables. aspx.

López, R. Ponce, G. Salazar, T (2018) "Eventos adversos en pediatría y medicamentos de alto riesgo". Enfermería Universitaria. Universidad Autónoma de México, ;8(3): 28-35.Recuperado de: http://revista-enfermeria.unam.mx/ojs/index. php/enfermeriauniversitaria/article/view/255

Martínez, C (2014) "Eventos adversos en el cuidado de enfermería brindado a niños hospitalizados". Revisalud. Universidad de Sucre, 2(1): 66-69. Recuperado de: https://revistas.unisucre.edu.co/ index.php/revisalud/article/view/133

Martínez, C. Álvarez, F. Garzón, C. Rave, I (2019) "Eventos adversos en niños hospitalizados: un desafío para el Cuidado de Enfermería". Revista ciencia y cuidado. 16(1):107-119.Recuperado de: https://revistas.ufps.edu.co/index.php/ cienciaycuidado/article/view/1547

Martínez, G. Gonzáles, C. Santos, J (2018) "Análisis del contenido de los reportes de eventos adversos registrados por estudiantes de pregrado durante su entrenamiento hospitalario en México". Revista Médica Sanitas., 21(2). Recuperado de: https:// www.researchgate.net/publication/327739539_ Analisis_del_contenido_de_los_reportes_de_eventos_adversos_registrados_por_estudiantes_ de_pregrado_durante_su_entrenamiento hospitalario_en_Mexico

Martínez, C (2018). Factores Relacionados con los Eventos Adversos por Medicamentos en Pediatría: Revisión. Quintero, H. Cuidarse es vida. Medellín: SedUnac. Recuperado de:
Ministerio de Salud de Colombia. Mejorar La Seguridad En La Utilización De Medicamentos [consultado 2019 feb 23] Recuperado de: https://www. minsalud.gov.co/sites/rid/Lists/BibliotecaDigital/ RIDE/DE/CA/seguridad-en-la-utilizacion-demedicamentos.pdf.

Milutinović, D. Simin, D. Zec, D (2015) "Factores de riesgo de la flebitis: un estudio con cuestionario de la percepción de las enfermeiras". Revista Latino-Americana. Enfermagem, 23(4): 677684. Recuperado de: http://www.scielo.br/ scielo. php? script=sci arttext \&pid=S0104$11692015000400677 \&$ Ing=en\&nrm=iso\&tlng=es

Monroy, S. Nagles, N (2014). Evaluación del programa uso seguro de medicamentos en un hospital especializado en pediatría y ortopedia infantil Bogotá D.C., 2012 -2013. (Tesis no publicada. Bogotá: Universidad Colegio Mayor De Nuestra Señora Del Rosario). Recuperado de: http:// repository.urosario.edu.co/handle/10336/8986

Nightingale F. Notas sobre Enfermería (1990). Madrid. Elsevier Masson.. Recuperado de; https://books. google.es/books?id=nSqzXx6jNEEC\&printsec=fro ntcover\&hl=es\#v=onepage \&q\&f=false

Organización Mundial de la Salud. [Online] [consultado 2019 Marzo 1] Recuperado de: https://www.who. int/mediacentre/news/releases/2007/pr51/es/.

Piñero, R (2014) “Errores de medicación en pediatría: en busca de una nueva vacuna". Anales de pediatría. Asociación Española de pediatría, 81(6): 241-242. Recuperado de: https://www.analesdepediatria. org/es-errores-medicacion-pediatria-busca-unaarticulo-S169540331400424X

Rendón, M. Restrepo, S. García, J (2019) "Determinación de la frecuencia, causas y evitabilidad de los eventos adversos en el servicio de pediatría de un IPS Quindio 2014" . Revista de investigaciones universidad del Quindio, 28(1): 4355.Recuperado de: http://blade1.uniquindio.edu. co/uniquindio/revistainvestigaciones/adjuntos/pdf/ e0f7_43\%20-\%2055.pdf

Riquelme, G (2012) "El cuidado de Enfermería: una reflexión sobre el cuidado humanístico". Horizonte de Enfermería. Pontificia Universidad Católica de Chile, 23 (2): 9-12. Recuperado de: http://horizonteenfermeria.uc.cl/images/pdf/23-2/ editorial.pdf.pdf

Riquelme, G. Ourcilleón, A (2013) "Descripción de eventos adversos en un hospital pediátrico de la ciudad de Santiago, Chile". Enfermería Global. Universidad de Murcia, 12(29):262-73.Recuperado de: http://scielo.isciii.es/scielo.php?script=sci_artte xt\&pid=S1695-61412013000100013 
Rodríguez, M. Sánchez, L. Jiménez, L (2017) "Eventos adversos, centinela y cuasifallas en el Servicio de Pediatría de un hospital de tercer nivel". Enfermedades Infecciosas y Microbiología Clínica. 37 (2):56-64.Recuperado de: https://www. medigraphic.com/pdfs/micro/ei-2017/ei172e.pdf

Sakuma, M. Ida, H. Nakamura, T. Ohta, Y. Yamamoto, K. Seki, S (2014) "Adverse drug events and medication errors in Japanese paediatric inpatients: a retrospective cohort study". Biomedics Journal Quality Safety, 23:830-837.Recuperado de: https:// qualitysafety.bmj.com/content/23/10/830.short.

Soto, M (2016). Errores de medicación relacionados con el paciente pediátrico. Congresos de enfermería Recuperado de: http://congresoenfermeria.es/ libros/2016/sala6/6729.pdf

Stacey, S. Coombes, I. Wainwright, C. Klee, B. Miller, H. Whitfield, K (2014) "Characteristics of adverse medication events in a children's hospital". Journal Paediatric Child Health, 50 (12):966-971. Recuperado de: https://www.ncbi.nlm.nih.gov/ pubmed/25049060

Suntasig, L(2015). Análisis de los errores de medicación y sus factores condicionantes en pacientes hospitalizados en los servicios de pediatría y neonatología del Hospital Un Canto a la Vida en el período comprendido entre los meses de enero a junio del año 2015". (Tesis no publicada. Quito: Pontificia Universidad Católica). Recuperado de: http://repositorio.puce.edu.ec/bitstream/ handle/22000/9740/Tesis\%20MS\%20FINAL \%20 FINAL\%20FINAL\%20NOVIEMBRE\%20-\%20 copia.pdf?sequence=1\&isAllowed=y
Teles, L. Figueiredo, A. Rocha, R. Marques, F (2019) "Characterization of adverse drug events identified by trigger in Brazilian pediatric inpatients". Journal de Pediatría. Sociedad Brasilera de Pediatría, 95(1):661-6868. Recuperado de: https://www.sciencedirect.com/science/article/pii/ S0021755718309690

Valdés, I. Duarte, M (2017). Capacidad hospitalaria de atención de eventos serios a medicamentos en población pediátrica Colombia 2010-2016. (Tesis de grado no publicada. Bogotá: Universidad De Ciencias Aplicadas y Ambientales). Recuperado de: https://repository.udca.edu. co/bitstream/11158/801/1/Capacidad $\% 20$ Hospitalaria\%20EAM\%20Pediatr\%C3\%ADa\%20 2010-2016.pdf

Vergara, T. Fica, A (2015) "Estudio de costo de las infecciones del torrente sanguíneo asociadas a catéter vascular central en pacientes adultos en Chile". Revista chilena de infectología. Sociedad Chilena de Infectología, ; 32( 6 ): 634638. Recuperado de: https://scielo.conicyt.cl/ scielo.php?script $=$ sci_abstract $\&$ pid $=$ S0716$10182015000700004 \& \operatorname{lng}=$ es\&nrm=iso

Woo, Y. Kim, H. Chung, S. Parque, B (2015) "Informes de errores de medicación pediátrica en la base de datos del sistema de informes de eventos adversos de Corea, 1989-2012: comparación con informes de adultos". Journal Korean Medicine Science.

Recibido: 4 de julio Aceptado: 10 de setiembre 